\title{
Nonlinear coupling effects of waves in a panda ring
}

\section{Preecha P. Yupapin}

Advanced Studies Center, Department of Physics, Faculty of Science, King Mongkut's Institute of Technology Ladkrabang (KMITL), Bangkok 10520, Thailand

\section{Email address:}

kypreech@kmitl.ac.th (P. P. Yupapin)

\section{To cite this article:}

Preecha P. Yupapin. Nonlinear Coupling Effects of Waves in A Panda Ring, Science Discovery. Vol. 1, No. 1, 2013, pp. 1-5. doi: 10.11648/j.sd.20130101.11

\begin{abstract}
All forms of waves in circular motions in circular ring resonator have shown the interesting behaviors, especially, in the nonlinear wave tracks, where the waves such as gravitation wave, sound wave, electromagnetic wave, matter wave and water wave can produce the whispering gallery modes (WGMs) with some certain conditions. This paper presents the use of a circular resonator (ring resonator) which is coupled by two nonlinear side rings known as a PANDA ring, in which the input waves can be in the forms of soliton, Gaussian pulses, photon or matter wave and introduced into the system. The WGMs can be generated due to the coupling effects of the two nonlinear side rings with the center ring and tunneling effects, which have shown many interesting results and aspects. Some expected outputs can be in the forms of surface plasmons, potential wells, leaky modes of waves, whispering gallery modes, matter waves (photons or particles). The use of WGMs for water wave and Rabi oscillation of photons (particles) in a PANDA ring are also discussed.
\end{abstract}

Keywords: Wgms, Nonlinear Wave Motion, Small Scale Optics; Interdisciplinary Science

\section{Introduction}

Generally, nonlinear behaviors in nature have been recognized as penalty rather than benefit of nature. However, it is not always true in particular when the penalty can be tuned to be benefit, for instance, when the whispering gallery modes (WGMs) of waves have shown the interesting results which can be useful for fundamental studies and applications, especially, in nanophysics, optoelectronics and nano-electronics. Particularly, after the announcement of Nobel Prize 2012 in Physics on the WGMs [1, 2], where the authors have confirmed that atoms can be trapped (stopped) using the WGM in a microsphere. However, there are two more kinds of devices that can be used to trap light beams (atoms), the use of microcavity arrays performed by Yanik and Fan [3], and a nonlinear microring resonator by Yupapin and Pornsuwancharoen [4] for stopping light. Recently, Ang and Ngo [5] have also done experiment to slowing the light in micro-resonators using a microring system. In this article, a new design of a nonlinear microring resonator is proposed which can be used to generate the WGMs in several forms. In particular case, the proposed device is made up of silica and $\operatorname{In} G a A s P / \operatorname{InP}$ with linear optical add-drop filter incorporating two nonlinear micro/nano rings on both sides of the center ring (modified add-drop filter). This particular configuration is known as a "PANDA" ring resonator
[6] as shown in Fig.1. Light pulse, for instance, Gaussian, bright and dark solitons are fed into the system through different ports such as add port and through port. By using the practical device parameters, the simulation results are obtained using the Optiwave and MATLAB programs and shown the interesting behaviors. The proposed system can be used for many applications, especially, when the device is coated by a metallic material [7]. By using the WGMs concept, several applications can be performed by a single device. In this article the use of WGMs for trapping tools, optical antenna, conjugate mirror, biomedical sensors and wave energy is discussed.

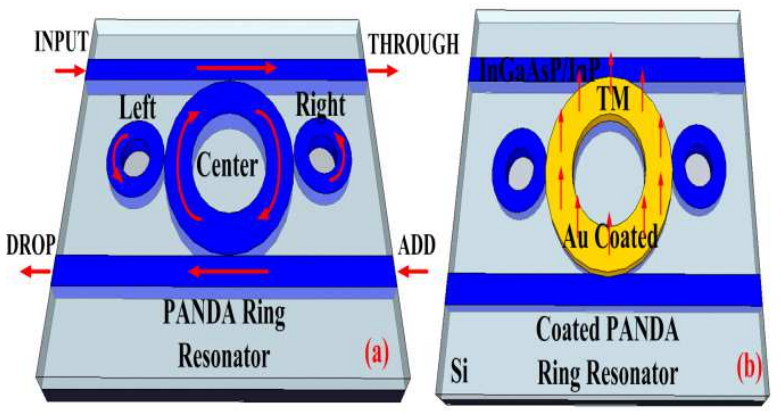

Figure 1. A conventional PANDA ring planar wave guide was named and designed by Uomwech et al [9] and Gold coated PANDA ring resonator for TM polarized coupling device [10] 


\section{Whispering Gallery Modes (WGMs)}

A PANDA ring resonator is as shown in Fig. 1, where the electromagnetic field in the form of WGM can be described by time-dependent Maxwell's equations and written as [8]

$$
\nabla^{2} \vec{E}-\frac{n^{2} \partial^{2} \vec{E}}{c^{2} \partial t^{2}}=0
$$

where $\mathrm{n}$ is the refractive index of the media and $\mathrm{c}$ is the speed of light in free space. For time-harmonic fields such as

$$
\vec{E}(\vec{r}, t)=\vec{E}(\vec{r}) e^{-i \omega t}
$$

Equation (1) is rewritten as:

$$
\nabla^{2} \vec{E}+\frac{n^{2} \omega^{2}}{c^{2}} \vec{E}=0
$$

When the resonance ring is placed in the $(\mathrm{x}, \mathrm{y})$ plane as shown in Fig. 1 and the electric field is polarized along the $\mathrm{z}$ direction, the transverse electric (TE) field is given by

$$
\vec{E}(\vec{r})=E(x, y) \vec{z}
$$

In this case, the Equation (2) becomes a scalar Helmholtz equation, which is given by

$$
\nabla^{2} \vec{E}+n^{2} k_{0}^{2} \vec{E}=0
$$

where $k_{0}$ is the free-space wave number.

For simplicity, the resonance ring is placed on the same plane as the waveguide. Thus, the WGM can be treated in a two-dimensional model. For outside boundaries, the scattering boundary condition is applied with initial amplitude of $E_{0}=0$. For the laser excitation source, the scattering boundary condition is also used with input amplitude of $E_{0}=1 \mathrm{~V} / \mathrm{m}$. The scattering boundary condition is commonly used to specify a boundary, which is transparent for a scattered wave and for an incoming plane wave. The Equation (3) as $\left(\nabla^{2}+k_{0}^{2}\right) E_{z}=0$ in $\mathrm{z}$ direction, can be written in cylindrical coordinates as

$$
\left(\frac{\partial^{2}}{\partial r^{2}}+\frac{1}{r} \frac{\partial}{\partial r}+\frac{1}{r^{2}} \frac{\partial^{2}}{\partial \varphi^{2}}+k_{0}^{2}\right) E_{z}(r, \varphi)=0
$$

where $r$ is the radial distance, $\varphi$ is the angle measured counterclockwise from the polar axis to the ray from the origin. By using the separation method, Equation (4) can be separated and split into two equations (i) radial and (ii) azimuthal components. An integer $m$ is introduced which is connected the two equations and corresponded to the number of optical cycles. The azimuthally equation is given as

$$
\left(\frac{\partial^{2}}{\partial \varphi^{2}}+m^{2}\right) E_{z}(\varphi)=0
$$

and solution of this equation is the complex exponentials, which is given by $E_{z}(\varphi)=e^{ \pm i m \varphi}$. The radial equation is the Bessel's function, which is given as

$$
\left(\frac{\partial^{2}}{\partial \varphi^{2}}+\frac{1}{r} \frac{\partial}{\partial r}+k_{0}^{2}-\frac{m^{2}}{r^{2}}\right) E_{z}(r)=0
$$

The solutions of Bessel's equations are the Bessel's function of the first $\mathrm{Jm}$ and the second Ym kinds, respectively. As the second function is singular at the origin, only the first kind function is retained inside the ring, whereas outside the ring both functions are well behave and must be retained. The Hankel functions are linear superposition of two Bessel function solutions corresponding to outward, $H_{m}^{1}=J_{m}+i Y_{m}$ and inward $H_{m}^{2}=J_{m}-i Y_{m}$ propagating cylindrical waves. The analysis of wave arriving at the resonator from the radial horizon is not considered here, and thus only the Hankel function of the first kind, $H_{m}^{1}$ is retained. Thus, the appropriate solution for the radial field dependence for both interior $(r<R)$ and exterior $(r>R)$ to the dielectric ring are given as

$$
\begin{aligned}
& E_{z}(r<R)=A_{m} J_{m}\left(\bar{k}_{1} r\right) \\
& E_{z}(r>R)=B_{m} H_{m}^{1}\left(\bar{k}_{2} r\right)
\end{aligned}
$$

Here $\bar{k}_{1}$ and $\bar{k}_{2}$ are complex propagation constants.

The complete axial electric field for interior and exterior to the ring is constructed from the azimuthal and radial solutions including the boundary condition at the interface $(r=R)$ which forces the tangential electric field to be continuous:

$$
\begin{gathered}
E_{z}(r, \varphi)=A_{m} J_{m}\left(\bar{k}_{1} r\right) e^{i( \pm m \varphi-\omega t)} \\
E_{z}(r, \varphi)=A_{m} \frac{J_{m}\left(\bar{k}_{1} R\right)}{H_{m}^{1}\left(\bar{k}_{2} R\right)} H_{m}^{1}\left(\bar{k}_{1} r\right) e^{i( \pm m \varphi-\omega t)}
\end{gathered}
$$

In this article, we introduce the PANDA ring resonator as shown in Fig. 2. Theoretical review for this concept is presented as following: the relative phase of the two output light signals after coupling into the optical coupler is $\pi / 2$, the signals coupled into the drop port and the through port obtained a phase difference of $\pi$. with respect to the input port signal. The input and control fields at the input port and control port are described as follows [7].

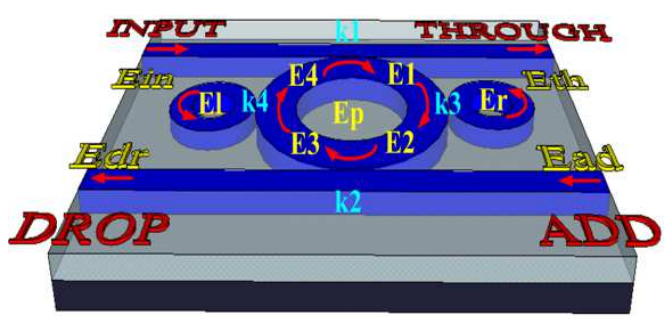

Figure 2. A Schematic of PANDA ring resonator. 


$$
\begin{gathered}
E_{T h}=x_{1} y_{1} E_{i n}+\left(j x_{1} x_{2} y_{2} \sqrt{\kappa_{1}} E_{4} E_{l} E_{1}-x_{1} x_{2} \sqrt{\kappa_{1} \kappa_{2}} E_{R} E_{a d}\right) e^{-\frac{\alpha}{2} \frac{L}{2}-j k_{n} \frac{L}{2}} \\
E_{D r}=x_{2} y_{2} E_{a d}+j x_{2} \sqrt{\kappa_{2}} E_{r} E_{1} e^{-\frac{\alpha}{2} \frac{L}{2}-j k_{n} \frac{L}{2}}
\end{gathered}
$$

Where $\quad x_{1}=\sqrt{1-\kappa_{1}} \quad, \quad y_{1}=\sqrt{1-\gamma_{1}} \quad, \quad x_{2}=\sqrt{1-\kappa_{2}}$ ， $y_{2}=\sqrt{1-\gamma_{2}}, \kappa_{1 \sqsubset}$ and $\kappa_{2}$ is the intensity coupling coefficient, $\gamma_{1}$ and $\gamma_{2}$ is the fractional coupler intensity loss, $\alpha$ is the attenuation coefficient, $\kappa_{n}=2 \pi / \lambda$ is the wave propagation number, $\lambda$ is the input wavelength light field and $\mathrm{L}=2 \pi \mathrm{Rad}$, where $\mathrm{Rad}$ is the radius of add-drop device. The circulated light fields, $E_{l}$ and $E_{r}$ are the light fields circulated components of the nanoring radii, $R_{l}$ and $R_{r}$ which are coupled to the left and right sides of the add-drop optical multiplexing system, respectively. The light field is transmitted and circulated in the right nanoring.

The characteristic lengths of surface plasmon polaritons, namely the SPPs wavelength ( $\lambda \mathrm{spp})$, propagation length (Lspp) and confinement to the surface (Lz) can be derived from the SPPs complex wavenumber, which is given as

$$
\kappa_{s p p}=\frac{\omega}{c} \sqrt{\frac{\varepsilon_{c} \varepsilon_{d}}{\varepsilon_{c}+\varepsilon_{d}}},
$$

where $\omega$ is the angular frequency, c the speed of light in vacuum, and $\omega_{c}$ and $\omega_{d}$ are the relative permittivity of the conductor and the dielectric respectively. For simplicity, the vacuum is considered as the dielectric with $\varepsilon_{d}=1$. The relative permittivity of the conductor is a complex quantity, $\mathcal{E}_{c}=\mathfrak{R}\left(\varepsilon_{c}\right)+i \mathfrak{I}\left(\varepsilon_{c}\right)$, which can be approximated by a Drude model for free charge carriers as [8]

$$
\varepsilon_{c}=\varepsilon_{\infty}\left(1-\frac{\omega_{P}^{2}}{\omega^{2}+i \gamma \omega}\right),
$$

where $\varepsilon_{d}$ is the high-frequency permittivity, $\gamma$ is the average collision rate of the charge carriers and $\omega_{P}=\sqrt{N e^{2} / \varepsilon_{\infty} \varepsilon_{0} m^{*}}$ is the plasma frequency. The waveguide charge carrier concentration is given by $\mathrm{N}$, while $e$ is the fundamental charge, $\varepsilon_{0}$ the vacuum permittivity and $m^{*}$ is the charge effective mass. Therefore, the use of SPPs mode coupling has to be confined well for TM mode in PANDA ring resonator.

\section{Simulation Results}

The whispering gallery mode result is obtained by using the Optiwave program as shown in Fig. 3. The ring material is InGaAsP/InP, where the device parameters are given in figure caption. By using the MATLAB program, the whispering gallery modes of four state of light i.e. fast, slow, stopping and storing can be generated and controlled simultaneously on-chip as shown in Fig. 4. The storing stage can be seen easily, while the stopping condition can be observed by satisfying following conditions: (i) the center signal is lost in time between fast and slow signals or (ii) there is no movement among trapped particles or molecules i.e. the exchange of angular momentum introduces the conservation of angular momentum, where the combination of scattering and gradient forces is balanced under the adiabatic process

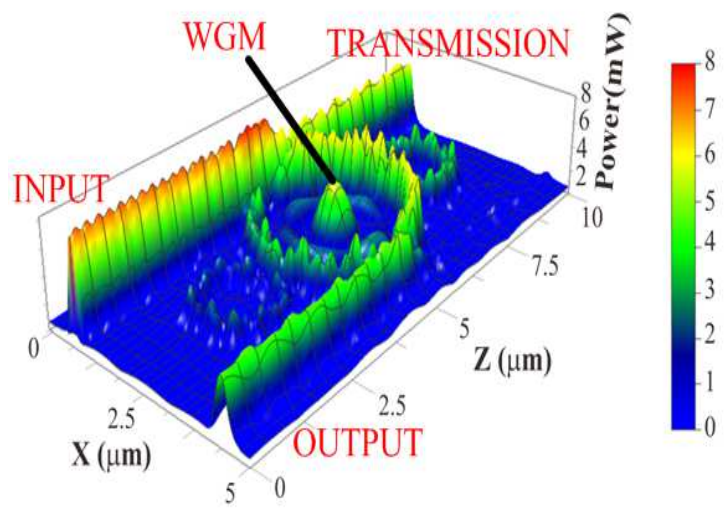

Figure 3. Result of whispering gallery mode of light within a PANDA ring waveguide InGaAsP/InP, $R_{1}=R_{2}=0.775 \mu \mathrm{m}, R_{a d}=1.565, A_{\text {eff }}=0.3 \mu \mathrm{m}^{2}$, $n_{\text {eff }}=3.14, n_{2}=1.3 \times 10^{-13} \mathrm{~cm}^{2} / W, \kappa_{l}=\kappa_{2}=\kappa_{3}=\kappa_{4}=0.5, \gamma=0.01, \lambda_{0}=$ $1,550 \mathrm{~nm}$

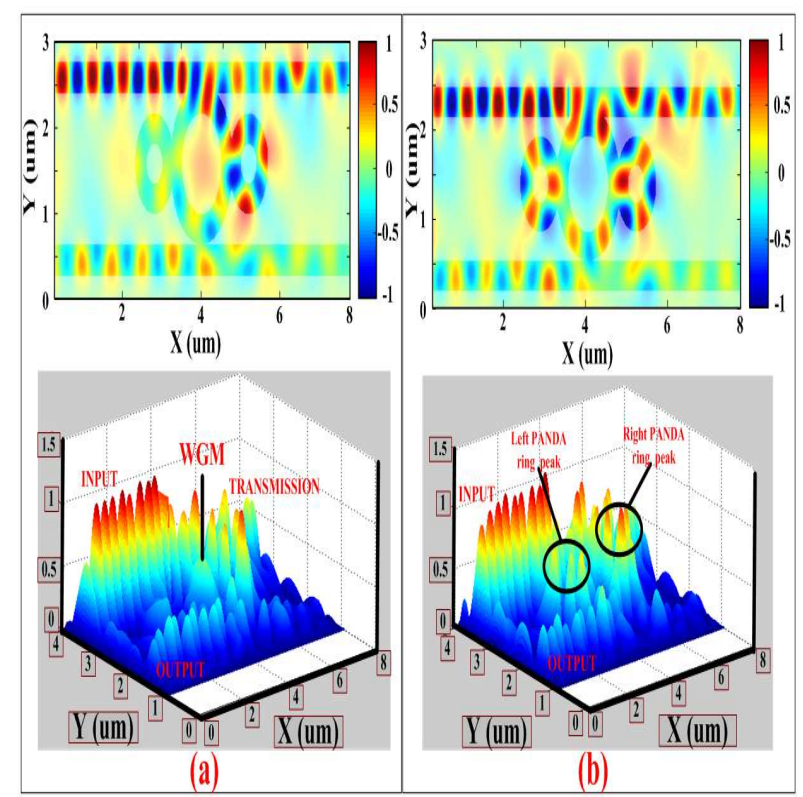

Figure 4. Stopping and storing light simultaneously detected using a $P A N D A$ ring, where (a) Center ring, (b) Side rings.

The stopping light in term of signal condition can be easily performed using the whispering gallery mode concept, where the fast and slow light can be used as the upper and lower time frames or upper side and lower side peak signals for the storing light at the center as shown in Fig. 4, where in this case the movement (modulated signals) longer than 150 fs, i.e. ms, ns, ps is observed (stopped). The input pulse is a Gaussian pulse with pulse width of $100 \mathrm{fs}$, the fast and slow 
time interval is known, however, the whispering gallery modes can be seen only under the resonant condition.

In this article, the full-wave finite-difference time-domain (FDTD) method is employed to solve Maxwell's equations. The FDTD modeling gives a useful design role, which is the combination of the propagation, scattering, diffraction, reflections, and polarization effects. It also handles well the material anisotropy, dispersion and nonlinearities without any pre-assumption of field behavior as the slowly varying amplitude approximation [9]. When a two dimensional (2-D) problem is included, the photonic device is laid out in the $\mathrm{X}-\mathrm{Z}$ plane, the propagation is along the $\mathrm{Y}$-axis, and the $\mathrm{Z}$ direction is assumed to be infinite and there is no variation in the Z-direction. This assumption removes all the $\partial / \partial z$ derivatives from Maxwell's equations and splits them into two (TE and TM) independent sets of equations. Because the field distribution is symmetrical about $\mathrm{z}=0$ plane, we only present the field distribution at $\mathrm{z}>0$, where we find that the field distribution oscillates in the cladding layer for TE mode, but is still confined well for TM mode. So, the mode coupling between TE WGM and the vertical propagating mode in the PANDA induces the radiation loss and a low Q-factor for TE WGMs [11]. Although, the mode wavelengths of TM WGMs are usually larger than the cut-off wavelengths of the radiated $\mathrm{HE}$ and EH modes with the same azimuthal and radial mode numbers in the microcylinder, so the corresponding mode-coupling radiation loss is absent for the TM WGMs. In our FDTD simulation, the perfectly matched layer (PML) absorbing boundary conditions applied by Berenger [12] and Yee scheme [13], which absorb the electromagnetic wave without any reflection at the computational boundary. A 100 fs-Gaussian pulse modulated by a $200 \mathrm{THz}$ carrier is exited. The vertical waveguide thickness and material composition is accounted by computing the effective refractive index $n_{\text {eff }}$ for the fundamental mode at $\lambda=1.55 \mu \mathrm{m}$ and then using $n_{\text {eff }}$ as the bulk material index of the core in the two dimensional simulations. In the vertical direction, each waveguide structure is $0.45 \mu \mathrm{m}$ thick, vertical core thicknesses of $0.3 \mu \mathrm{m}$ to $0.5 \mu \mathrm{m}, n_{\text {eff }}$ is between 3.2 to 3.4 , in which the parameters are obtained by using the related practical material parameters of InGaAsP/InP. Here, the waveguide core $n=3.14$ is bordered on each side by air $n=1$. The parameters for add-drop optical multiplexer and both nanorings on the left and right hand sides of the PANDA ring are set at $\mathrm{Rl}=\mathrm{Rr}=0.775 \mu \mathrm{m} \mu \mathrm{m}$ and radius of the center ring is $\mathrm{Rad}=1.56 \mu \mathrm{m}$. The coupling coefficient ratios are $\kappa 1=\kappa 4=0.5, \kappa 2=\kappa 3=0.5$, effective core area of the waveguides is $A_{\text {eff }}=0.25 \mu \mathrm{m} 2$, and waveguide loss coefficient is $\alpha=0.1 \mathrm{~dB} / \mathrm{mm}$.

In this case the modulated signal is required to switch off the whispering gallery mode power via the add port, where atoms/molecules at the device center can be trapped and transported along the wave guide by the surface plasmon tweezers as shown in Fig. 5. The dynamic tweezers are generated by a PANDA ring, where in practice, particle angular momentum can be introduced by a metal coating material on the waveguide surface or combining the external modulation via the add port, which can be used to trap and transport atom/molecule to the required destination when the gradient force is greater than the scattering force along the waveguide. More applications such as the use of Rabi oscillation for optical antenna [14, 15], conjugate mirror for $3 \mathrm{D}$ display and imaging, biomedical sensors and wave energy for home generator use are also the interesting aspects of investigations.
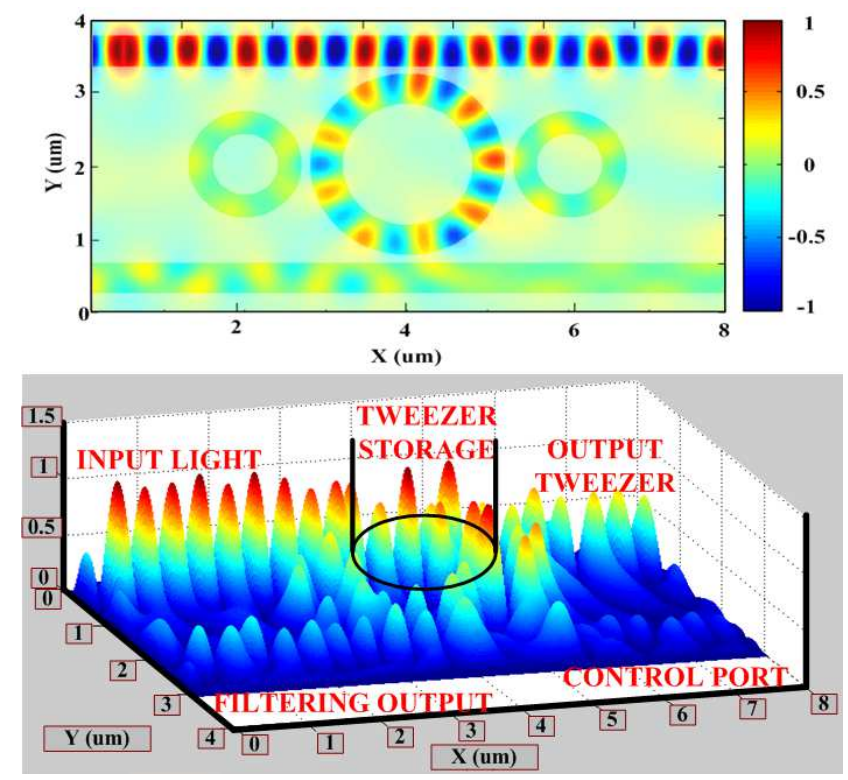

Figure 5. Dynamic tweezers are generated by a PANDA ring and transmitted via a Through port for atoms/molecules harvesting and transportation.

\section{Conclusion}

Forms of light in a PANDA ring resonator have been manipulated, and it has been observed that the two nonlinear side rings have shown the interesting results and aspects. The input light can be in the forms of soliton or Gaussian pulses. The use of photon or matter wave as input is also possible, in which the matter wave concept is available within the PANDA ring waveguide. The expected output light can be in the forms of surface plasmon, potential wells, leaky modes, whispering gallery modes, matter wave and photons (particles). The use of a PANDA ring is also available for water wave and Rabi oscillation which can be used to form the water wave energy and optical antenna systems. Moreover, the trapped particles/ions by WGMs within a PANDA ring can be stored and formed the quantum fluid and antimatter material applications.

\section{Acknowledgments}

The author would like to give the acknowledgement to King Mongkut's Institute of Technology Ladkrabang (KMITL), Bangkok 10520, Thailand for the laboratory facilities. 


\section{References}

[1] N. Pornsuwancharoen and P.P. Yupapin, " Generalized fast, slow, stop and store light optically within a nanoring resonator", Microwave \& Optical Technology Letters, $51(4)(2009) 899-902$.

[2] D.J. Wineland, J.J. Bollinger, Wayne M. Itano, J.D. Prestage, "Angular momentum of trapped atomic particles", JOSA B, 2(11)(1985)1721-1730.

[3] J.C. Knight, N. Dubreuil, V. Sundoghdar, J. Hare, V. Lefevre-Seguin, J.M. Raimond and S. Haroche, "Characterizing whispering-gallery modes in microspheres by direct observation of the optical standing-wave pattern in the near field", Optics Letters, 21(10)(1996)698-670.

[4] M.F. Yanik and S. Fan, "Stopping and storing light coherently”, Physical Review Letters, 92(2004)083901-3.

[5] P.P. Yupapin and N. Pornsuwancharoen, "Proposed nonlinear microring resonator arrangement for stopping and storing light", IEEE Photonics Technology Letters, 21(6)(2009)404-406.

[6] T. Y. L. Ang and N. Q. Ngo, "Tunable flat-band slow light via contra-propagating cavity modes in twin coupled microresonators ", JOSA B, 29(5)(2012)924-933.

[7] T. A. Birks, J. C. Knight and T. E.Dimmick, "High-resolution measurement of the fiber diameter variations using whispering gallery modes and no optical alignment", IEEE Pho-

tonics Technology Letters, 12 (2000) $182-183$.

[8] K.S. Yee, "Numerical solution of initial boundary value problems involving Maxwell's equations in isotropic media", IEEE Transaction on Antennas Propagation, 14, 302-307 (1966).

[9] K. Uomwech, K. Sarapat and P.P. Yupapin, "Dynamic modulated Gaussian pulse propagation within the double PANDA ring resonator system", Microwave \& Optical Technology Letters, 52(8)(2010)1818-1821.

[10] N. Thammawongsa, N. Moonfangklang, S. Mitatha, P.P. Yupapin, "Novel nano-antenna system design using photonics spin in a panda ring resonator", PIER L, 31(2012) 75-87.

[11] E. Waks, V. Jelena, "Coupled mode theory for photonic crystal cavity-waveguide interaction", Optics Express, 13(13)(2005) 5064-5073.

[12] M. Pöllinger, D. O’Shea, F. Warken, A. Rauschenbeutel, "Ultrahigh-Q tunable whispering-gallery-mode microresonator”, Physical Review Letters, 103.(2009) 053901.

[13] L.-P. Berenger, "Perfectly matched layer for the FDTD solution of wave-structure interaction problem", IEEE Transaction on Antennas Propagation, 44 (1) (1996)110-118.

[14] Y. Hiroyuki and K. Ujihara, Spontaneous Emission and Laser Oscillation in Microcavities. Boca Rato, CRC Press., 1995, p. 6.

[15] V. Kerry, Optical Microcavities. Singapore: World Scientific Pub., 2004, p. 368. 